\title{
theoretical analysis of finite-height semiconductor-on-insulator based planar photonic crystal waveguides
}

\section{Søndergaard, Thomas; Arentoft, Jesper}

Published in:

Journal of Lightwave Technology

Link to article, DOI:

$10.1109 / \mathrm{JLT} .2002 .800380$

Publication date:

2002

Document Version

Publisher's PDF, also known as Version of record

Link back to DTU Orbit

Citation (APA):

Søndergaard, T., \& Arentoft, J. (2002). theoretical analysis of finite-height semiconductor-on-insulator based planar photonic crystal waveguides. Journal of Lightwave Technology, 20(8), 1619-1626.

https://doi.org/10.1109/JLT.2002.800380

\section{General rights}

Copyright and moral rights for the publications made accessible in the public portal are retained by the authors and/or other copyright owners and it is a condition of accessing publications that users recognise and abide by the legal requirements associated with these rights.

- Users may download and print one copy of any publication from the public portal for the purpose of private study or research.

- You may not further distribute the material or use it for any profit-making activity or commercial gain

- You may freely distribute the URL identifying the publication in the public portal 


\title{
Theoretical Analysis of Finite-Height Semiconductor-on-Insulator-Based Planar Photonic Crystal Waveguides
}

\author{
Thomas Søndergaard, Jesper Arentoft, and Martin Kristensen
}

\begin{abstract}
A planar photonic crystal waveguide based on the semiconductor-on-insulator (SOI) materials system is analyzed theoretically. Two-dimensional (2-D) calculations and comparison with dispersion relations for the media above and below the finite-height waveguide are used to obtain design guidelines. Three-dimensional (3-D) calculations are given for the dispersion relations and field profiles. The field profiles obtained using 2-D and 3-D calculations are qualitatively similar. However, we find that compared with 2-D calculations, the frequencies of the guided modes are shifted and the number of guided modes changes. The theoretically predicted frequency intervals, where the waveguide supports leakage-free guidance of light, are compared with an experimental measurement for propagation losses. Two out of three frequency intervals coincide with low-measured propagation losses. The poor guidance of light for the third frequency interval is explained theoretically by investigating the vertical localization of the guided modes.
\end{abstract}

Index Terms-Design, integrated optics, optical waveguide, photonic bandgap, photonic crystal.

\section{INTRODUCTION}

$\mathbf{I}$ N 1987, Yablonovitch and John suggested the possibility of making periodic dielectric structures in which light cannot propagate in any direction for certain frequency intervals [1], [2]. These frequency intervals are known as photonic bandgaps, and they are analogous to the electronic bandgaps in semiconductor materials that can be related to the periodic arrangement of atoms on a crystal lattice. The new dielectric materials are often referred to as photonic crystals. Since then, a new field of research has started that seeks to understand the new physics of these materials and to take advantage of the new material properties for making novel optical components [3], [4].

Photonic crystals can be incorporated in existing optical components with the purpose of improving their optical properties. An example is the vertical-emitting diodes, where it has been suggested that a photonic crystal material on top of the structure

Manuscript received September 24, 2001; revised April 8, 2002. This work was supported by the EU-project PICCO (photonic integrated circuits using photonic crystal optics).

T. Søndergaard was with COM, the Technical University of Denmark, DK-2800 Lyngby, Denmark. He is now with Micro Managed Photons, DK-9220 Aalborg, Denmark (ts@mmphotons.dk).

J. Arentoft was with COM, the Technical University of Denmark, DK-2800 Lyngby, Denmark. He is now with Sparkolor, Santa Clara, CA 95050-2704 USA.

M. Kristensen is with COM, the Technical University of Denmark, DK-2800 Lyngby, Denmark.

Digital Object Identifier 10.1109/JLT.2002.800380 improves the extraction efficiency [5]. Photonic crystals are also interesting for making novel cavities and lasers [6]-[9].

Components based on photonic crystals draw interest in use for integrated optics because it is possible to confine and manipulate light in a small spatial region. For example, it has been suggested that by using waveguides based on photonic crystals, it may be possible to obtain efficient transmission of light (>98\%) through sharp bends [10] and branching points [11]. These are important issues for integrated optics, where many optical components should be integrated together in a small spatial region. Waveguides are an essential ingredient for integrating optical components.

Previous theoretical work on photonic crystal waveguides has to a large extent been calculations for two-dimensional (2-D) waveguide structures [10]-[15]. For microwave frequencies, transmission through waveguides based on three-dimensional (3-D) photonic crystals has been investigated experimentally [16]. For optical frequencies, researchers presently focus on waveguides based on 2-D photonic crystals.

For finite-height 2-D photonic crystal waveguide structures with a relatively low vertical index contrast there has also appeared experimental work on transmission and propagation losses [17], [18], and there have been experimental demonstrations of features from 2-D calculations such as minibands [19]. When operated at frequencies in the bandgap, these waveguides are inherently lossy because the vertical index contrast is not sufficiently high to completely confine the light vertically. However, these losses can be low. Experimentally, losses as low as $11 \mathrm{~dB} / \mathrm{mm}$ and $20 \mathrm{~dB} / \mathrm{mm}$ have been reported [17], [18]. Modeling of these losses is pursued by a number of authors [20], [21].

In this paper, we investigate a photonic crystal waveguide design with a relatively high vertical index contrast. Theoretically, photonic crystal waveguides with a high vertical index contrast may support leakage-free bandgap guidance of light. For these waveguides, it is possible to obtain design rules for construction of leakage-free bandgap-guiding waveguides based on 2-D calculations [22], [23]. Also appearing in the literature are 3-D theoretical calculations for finite-height waveguides [24]-[28].

Finite-height waveguides with air above and below have been explored experimentally by Charlton et al. [29], [30] and Loncar et al. [31]. Waveguides where one seeks to confine light using a smaller vertical index step have also been explored experimentally by Tokushima et al., Chow et al., and Notomi et al. [32]-[34]. 


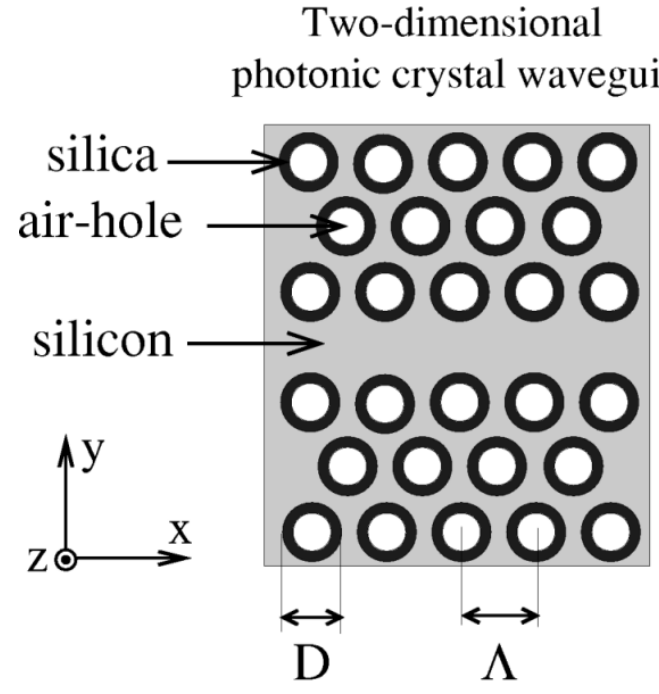

(a)

\author{
Finite-height \\ photonic crystal waveguide
}

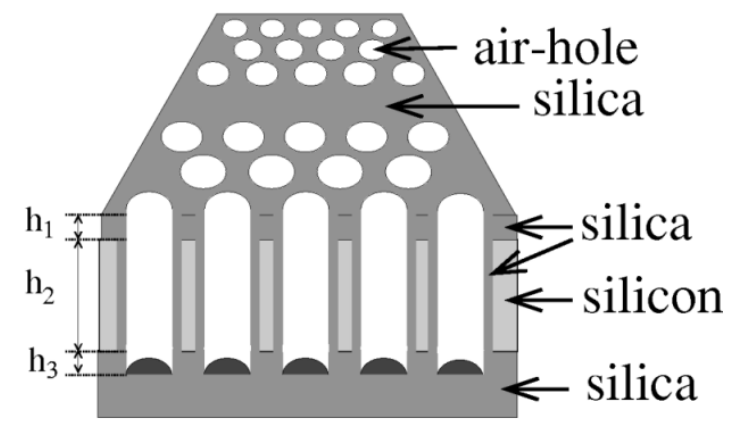

(b)

Fig. 1. (a) A 2-D planar photonic crystal waveguide. (b) The finite-height planar photonic crystal waveguide.

In this paper, we investigate theoretically a photonic crystal waveguide based on the semiconductor-on-insulator (SOI) materials system. We have previously developed a technique for obtaining design guidelines for photonic crystal waveguides based on 2-D calculations [22], [23]. In Section II, this technique is used for obtaining design guidelines for the photonic crystal waveguide.

For a specific structure that has been experimentally fabricated [35], [36], 3-D calculations are given in Section III for the dispersion relations and field profiles. The 3-D analysis is compared with the 2-D calculations. The 3-D calculations are further compared with an experimental measurement for propagation losses. The measurements can be explained by theoretically predicted frequency ranges for guidance of light and the calculated vertical localization of the guided modes. The consequences on confinement of light due to the waveguide design being asymmetric are obtained from an analysis of the vertical confinement of the electromagnetic mode fields. The conclusion is presented in Section IV.

\section{Design Guidelines Based on 2-D Calculations}

In this section, design guidelines will be given for the photonic crystal waveguide illustrated in Fig. 1. The structure of interest is the finite-height waveguide shown in Fig. 1(b). All calculations in this section will, however, be for the 2-D structure shown in Fig. 1(a). Design guidelines are obtained for the finite-height structure by comparing the 2-D calculations with dispersion relations for the media above and below the finiteheight waveguide structure.

Consider the 2-D photonic crystal waveguide shown in Fig. 1(a), where air holes are arranged on a triangular lattice in silicon (dielectric constant 12). We will further assume that there is a layer of silica (dielectric constant 2.1) on the inside of the walls of the air holes with a thickness of $0.125 \Lambda$, where $\Lambda$ is the lattice constant of the photonic crystal material. A line defect or a waveguide has been created in the photonic crystal material by removing a row of air holes. Because the photonic

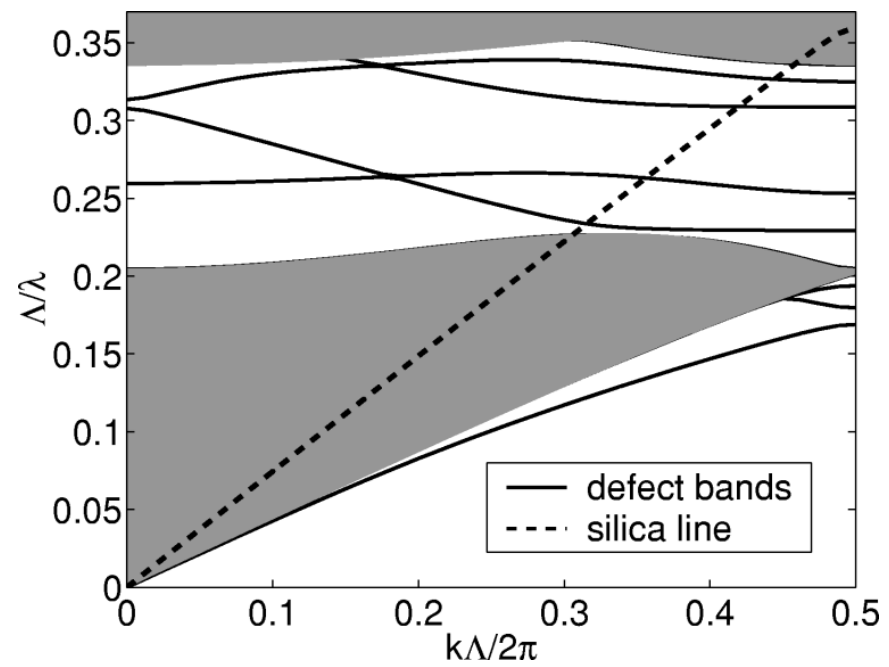

Fig. 2. Banddiagram for the 2-D planar photonic crystal waveguide.

crystal waveguide is a periodic structure, the electromagnetic fields can be expanded in Bloch modes on the form

$$
\mathbf{E}(\mathbf{r})=\mathbf{U}_{\mathbf{k}}(\mathbf{r}) \exp (i \mathbf{k} \cdot \mathbf{r})
$$

where $\mathbf{r}$ is the position and $\mathbf{U}_{\mathbf{k}}(\mathbf{r})$ is a function with the same periodicity as the periodic structure, and there is also a phase factor $\exp (i \mathbf{k} \cdot \mathbf{r})$ where $\mathbf{k}$ is the Bloch wave vector.

The banddiagram for the photonic crystal waveguide is shown in Fig. 2. All calculations presented in this paper have been calculated using plane-wave-expansion theory and a variational principle [37]. On the $x$ axis, we have the Bloch wave vector normalized relative to the crystal lattice constant $\Lambda$, and on the $y$ axis, we have shown the normalized frequency $\Lambda / \lambda$, where $\lambda$ is the free-space wavelength.

The banddiagram shows the allowed combinations of Bloch wave vector and frequency for TE-polarized light (electric field in the $x y$ plane). The shaded continuum corresponds to combinations of wave vector and frequency that are allowed in the 
photonic crystal material surrounding the waveguide, and this region does not correspond to the guided modes of the waveguide. However, the discrete defect bands correspond to solutions to Maxwell's equations where the fields are localized to the region of the waveguide and these bands represent the guided modes of the waveguide. Note that if a waveguide had not been introduced, the only change in the diagram would be that the discrete bands would not be there and there would be no modes allowed for the frequency range from $\Lambda / \lambda=0.227$ to 0.336 . This frequency interval is the photonic bandgap of the photonic crystal material. In this 2-D picture, the defect bands provideinformation on the frequency ranges where the waveguide supports guidance of light. The slope of the bands gives the energy velocity [11].

A real photonic crystal waveguide such as the one shown in Fig. 1(b) is not a 2-D structure, and it is necessary to take into account the effect of a real photonic crystal waveguide being a finite-height structure. In the finite-height photonic crystal waveguide [Fig. 1(b)], a slab of the 2-D photonic crystal waveguide is placed on silica. The air holes also extend into the silica. For the experimental structure [35], [36], silica is grown on top of the structure by thermal oxidation. This makes the structure more symmetric in the vertical dimension. Thermal oxidation also makes the silica grow on the inside of the air holes. In the thermal oxidation process, part of the silicon is converted into silica.

Information on the finite-height structure can be obtained from the banddiagram (Fig. 2) by plotting the dispersion curve, the silica line, for the silica material with air holes below the slab. All combinations of frequency and wave vector above this line are allowed in the silica material. Consequently, leakage-free confinement of light to the silicon slab in the vertical dimension is only possible if the waveguide structure is operated at a frequency and wave vector below the silica line. Fortunately, in our case there are four defect bands or guided modes that are below the silica line, and we can expect that leakage-free guidance of light is possible for the finite-height waveguide design for an appropriately chosen height of the silicon slab.

If the silica material is replaced by another material with a higher refractive index, it would be necessary to use another dispersion line instead with a smaller slope, and in that case, leakage-free guidance of light for frequencies in the bandgap is not possible if the bandgap is above such a dispersion line. Note that in the case where leakage-free confinement of light is not possible, the losses can still be low.

The position in frequency of the photonic bandgap is important, and this position depends on the size of the air holes $D$. Note that the silica line hits the edge of the banddiagram at a normalized frequency of 0.36 , and this frequency may be interpreted as an upper-frequency limit for leakage-free bandgap guidance of light. The upper-frequency limit $\Lambda / \lambda=0.36$ is specifically related to the diameter $D=0.75 \Lambda$, and in general, the upper-frequency limit will depend slightly on $D$.

In Fig. 3, this frequency limit has been plotted together with the bandgap as a function of the diameter of the holes $D$, and we can see that a large bandgap below the silica limit can be obtained by choosing $D=0.75 \Lambda$. Note that in the calculation

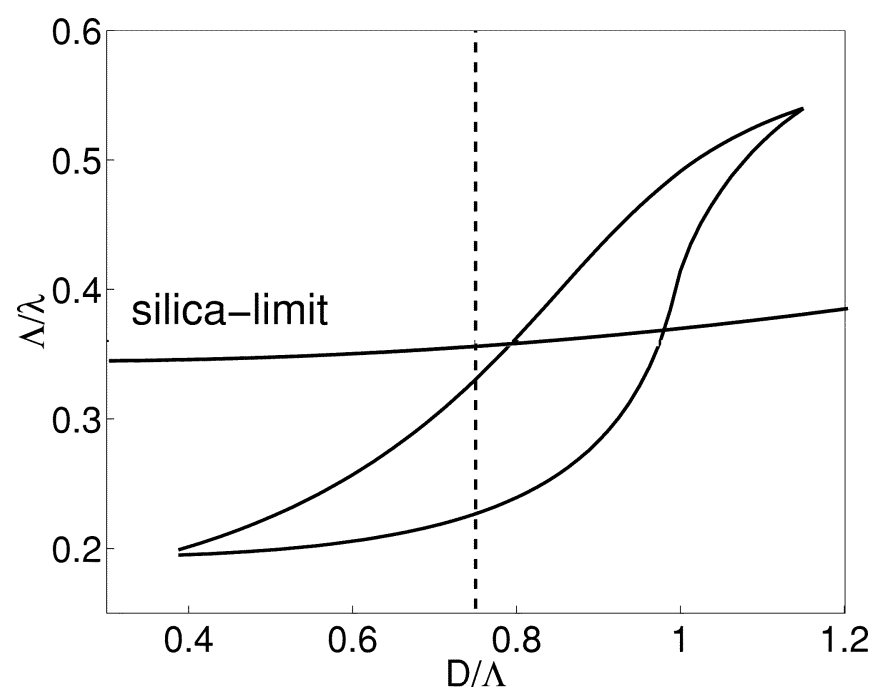

Fig. 3. Bandgap for the 2-D photonic crystal as a function of the diameter $D$ of the holes in the crystal.

shown in Fig. 3, we have taken into account the presence of the layer of glass on the inside of the air holes with a thickness of $0.125 \Lambda$. For structures where there is no layer of glass on the inside of the air holes, the air holes would overlap for $D>\Lambda$, and in that case, the bandgap for TE polarization would break down almost immediately as the hole diameter becomes $D=\Lambda$ (see e.g., [23]). In the present case, a bandgap exists for somewhat larger values of $D$ because there is a layer of glass on the inner walls of the holes.

The size of the air holes obtained from 2-D calculations $D=$ $0.75 \Lambda$ gives a design guideline for the experimental realization of finite-height waveguides.

To compare 2-D and 3-D calculations, for the 2-D photonic crystal waveguide, we have also calculated the amplitude of the magnetic field squared for the four bandgap-guided modes at $k \Lambda / 2 \pi=0.46$. These field profiles are shown as contour plots in Fig. 4.

\section{Three-Dimensional Theoretical Analysis}

In this section, the finite-height photonic crystal waveguide shown in Fig. 1(b) is analyzed by 3-D calculations. The size of holes, height of silicon slab, etc., used for the theoretical 3-D calculations have all been obtained from an experimentally fabricated structure [36]. This leaves no free parameters, and therefore, when we compare the theory with experimental propagation loss measurements later in this paper, note that no parameters have been tuned or fitted to obtain a better agreement. The height of the silicon slab is $h_{2}=0.6 \Lambda(240 \mathrm{~nm})$, the size of the holes is $D=0.75 \Lambda(300 \mathrm{~nm})$, and the thickness of the glass layer on the inside of the holes is $0.125 \Lambda(50 \mathrm{~nm})$.

The dimensions for the holes and glass layer on the inside is the same as for the 2-D calculations presented in the previous section. In the theoretical calculations, we assume that the glass layer with air holes on top of the structure is infinitely thick, i.e., $h_{1}=\infty$, and we also assume that the air holes are etched infinitely deep into the bottom cladding, i.e., $h_{3}=\infty$. In the real experimental structure, however, the heights are $h_{1}=50 \mathrm{~nm}$ and $h_{3}=100 \mathrm{~nm}$. 

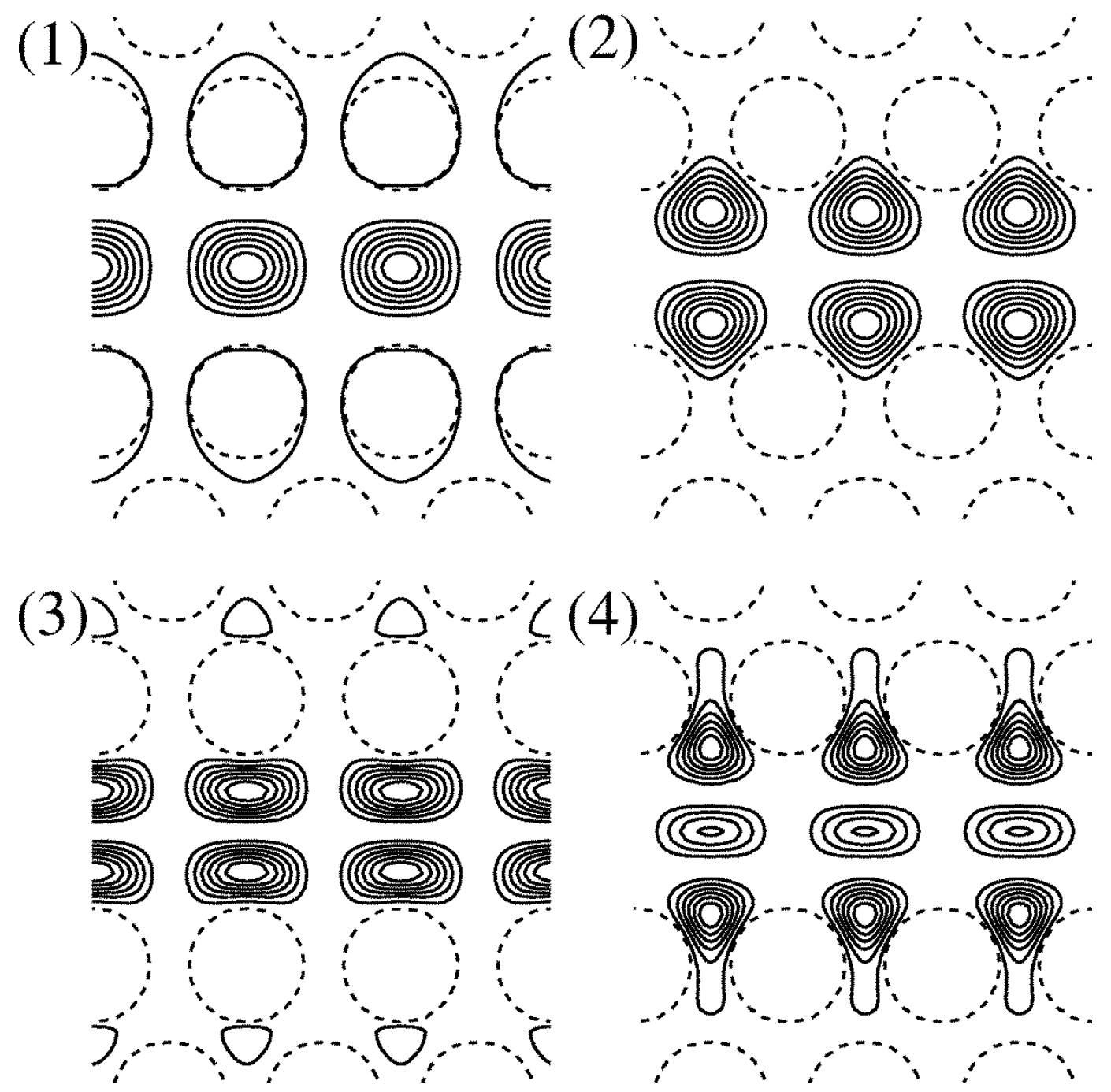

Fig. 4. Amplitude of magnetic field squared for the four bandgap-guided modes in a 2-D planar photonic crystal waveguide $(k \Lambda / 2 \pi=0.46)$.

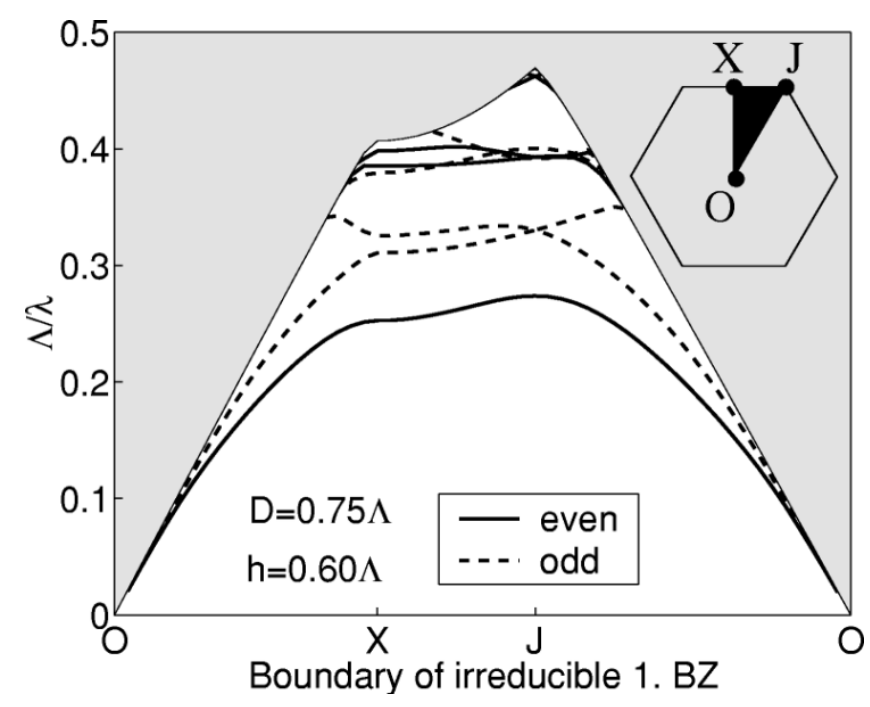

Fig. 5. Banddiagram for the finite-height photonic crystal slab.

The banddiagram for the finite-height photonic crystal slab without a line defect or a waveguide introduced is shown in Fig. 5. Here, the bandstructure is investigated for those Bloch wave vectors that are on the boundary of the irreducible Brillouin zone, shown as an inset in Fig. 5. The shaded region is the light cone for the silica material above and below the finiteheight photonic crystal slab. All combinations of wave vector and frequency in the shaded region are allowed in the silica material. Confinement of light to the slab requires that we are below the light cone. The modes below the light cone have been divided into even modes (solid lines) and odd modes (dashed lines). In the center plane of the slab, the even modes are polarized in the same way as TE modes for 2-D photonic crystals (the electric field is in the symmetry plane and the magnetic field is perpendicular to the symmetry plane). The odd modes correspond to TM polarization for the 2-D case. Outside this symmetry plane, there is polarization mixing so that, in the 3-D case, we often refer to TE-like modes (even) and TM-like modes (odd), instead of TE and TM, as was appropriate in the 2-D case.

Compared with the 2-D bandgap shown in Fig. 3, the bandgap has moved up in frequency. For example the lower edge of the TE gap in Fig. 3 is approximately $0.227 \Lambda / \lambda$, and in Fig. 5, the lower edge of the gap in even modes is approximately $0.274 \Lambda / \lambda$. Due to the finite height, the fields will be affected by the presence of a material with a lower refractive index 


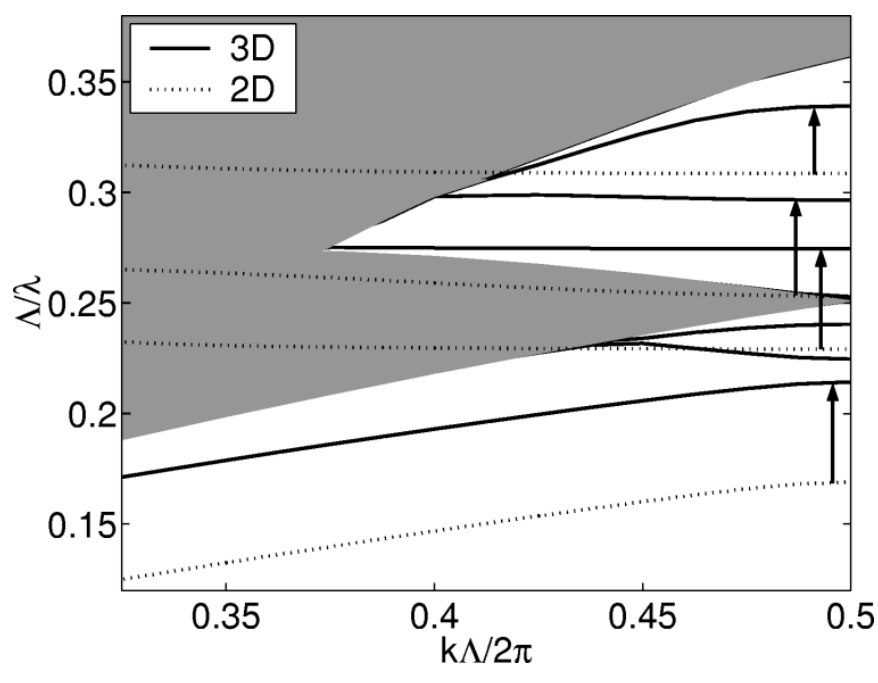

Fig. 6. Banddiagram for the finite-height photonic crystal waveguide (3D). Some dispersion curves obtained from a corresponding 2-D calculation are shown as dotted curves (2D), and arrows indicate the shift in frequency of dispersion curves when going from the 2-D to the 3-D calculation.

above and below the slab. A variational principle can be used for explaining that bands and bandgaps move up in frequency in such cases [3].

When a linear waveguide is introduced in the structure, the Brillouin zone collapses to a line interval, and the bands must be projected along one specific direction. The 3-D dispersion curves for the finite-height photonic crystal waveguide are shown in Fig. 6. The shaded regions now correspond either to a continuum of modes allowed in the photonic crystal slab surrounding the waveguide or to the continuum of modes allowed in the silica material above and below the waveguide. The diagram would be similar to Fig. 2 if the region above the silica line were shaded. By comparing with the corresponding 2-D calculation (Fig. 2), we see that there are now only three bandgap-guided modes, rather than four, as in the 2-D case. The frequencies have also been shifted on the order of 0.04 in normalized frequency units.

In order to emphasize this shift in frequency, some of the bands from Fig. 2 have been included as dotted curves (2D) in Fig. 6, and arrows indicate the shift when going from the 2-D calculation (2D) to the 3-D calculation (3D). If the height of the silicon layer in the photonic crystal waveguide is decreased, the dispersion relations tend to move up in frequency; if the height is increased, the dispersion relations tend to move down in frequency. The frequency shift, which is approximately 0.04 with the present height, can therefore be increased or decreased by choosing a different height.

The magnetic field squared through the center of the finite-height waveguide is shown for the three bandgap-guided modes in Fig. 7, and clearly, modes 1 and 2 appear almost identical to the result obtained using 2-D calculations. For the third band, however, some differences are easily seen by comparison, but the overall symmetry has been maintained. Of course, the change in the number of bandgap-guided modes from four to three and the shift in frequency are significant differences between the 2-D and 3-D analyses. Two-dimensional calculations are useful for making design guidelines and have the advantage of being computationally more manageable. Because of the differences between the 2-D and 3-D calculations, the 3-D calculations are necessary when comparing theoretical predictions with experiments to obtain good agreement. Two-dimensional calculations may be sufficient for structures with a low vertical index contrast.

From the banddiagram (Fig. 6), we will expect guidance of light for the frequency ranges covered by the three defect bands related to bandgap-guided modes. These frequency ranges have been plotted in Fig. 8 together with experimental propagation loss measurements for the photonic crystal waveguide. The loss measurement was obtained from five waveguides with different lengths, including a $90^{\circ}$ bend obtained by gradual rotation of the crystal lattice in the bend region. The waveguides with the bends are described in [35]. The propagation loss per unit length was obtained by comparing the transmission loss for the waveguides with different lengths. The details of the experimental work on propagation losses will be presented elsewhere in [36].

Almost exactly at the frequencies of the calculated bands 1 and 2, low propagation losses are observed in Fig. 8. The calculated bands 1 and 2 cover relatively narrow frequency ranges. The measured frequency ranges with low propagation losses are wide compared with the calculation. One possible reason for the difference in width can be slight variations in the size and position of air holes in the structure used for the measurement. A shift in the size of the air holes would result in a shift in frequency for the narrow frequency intervals with leakage-free guidance of light. Alternatively, it may again be noted that the losses are not necessarily high outside the narrow calculated bands.

From the view of the theoretical calculation, one possible drawback of bands 1 and 2 may be that the slope of the bands is small. The energy propagation velocity of the guided mode is proportional to the slope of the band, or equivalently, the effective modal refractive index is inversely proportional to the slope. Therefore, the energy velocity of modes in, e.g., an optical fiber or a ridge waveguide may be much higher compared with the energy velocity of the modes corresponding to bands 1 and 2 . The modal refractive index of the optical fiber or ridge waveguide is therefore relatively low compared with that of modes 1 and 2 . The impedance mismatch due to the difference in mode index may make coupling of light into the photonic crystal waveguide difficult. The slope of band 3 is relatively large compared with the slope of the bands 1 and 2, and consequently, coupling of light into the waveguide may be easier at the frequencies of band 3 due to the improved impedance match to the modes of, e.g., a ridge waveguide. Unfortunately, at the frequencies covered by the third band, some decrease is observed in the propagation losses, but the losses do not become low.

In order to understand why the losses for the third defect band are not low, we analyzed the vertical localization of the fields in the photonic crystal waveguide. The localization of the electric field squared is illustrated for the three bands in Fig. 9. The fields have been plotted for a plane defined by the waveguide axis and the out-of-plane direction or $z$ direction (see Fig. 1). We prefer to use the electric field here instead of the magnetic field because the fields for all three bands have high electric field intensity in 

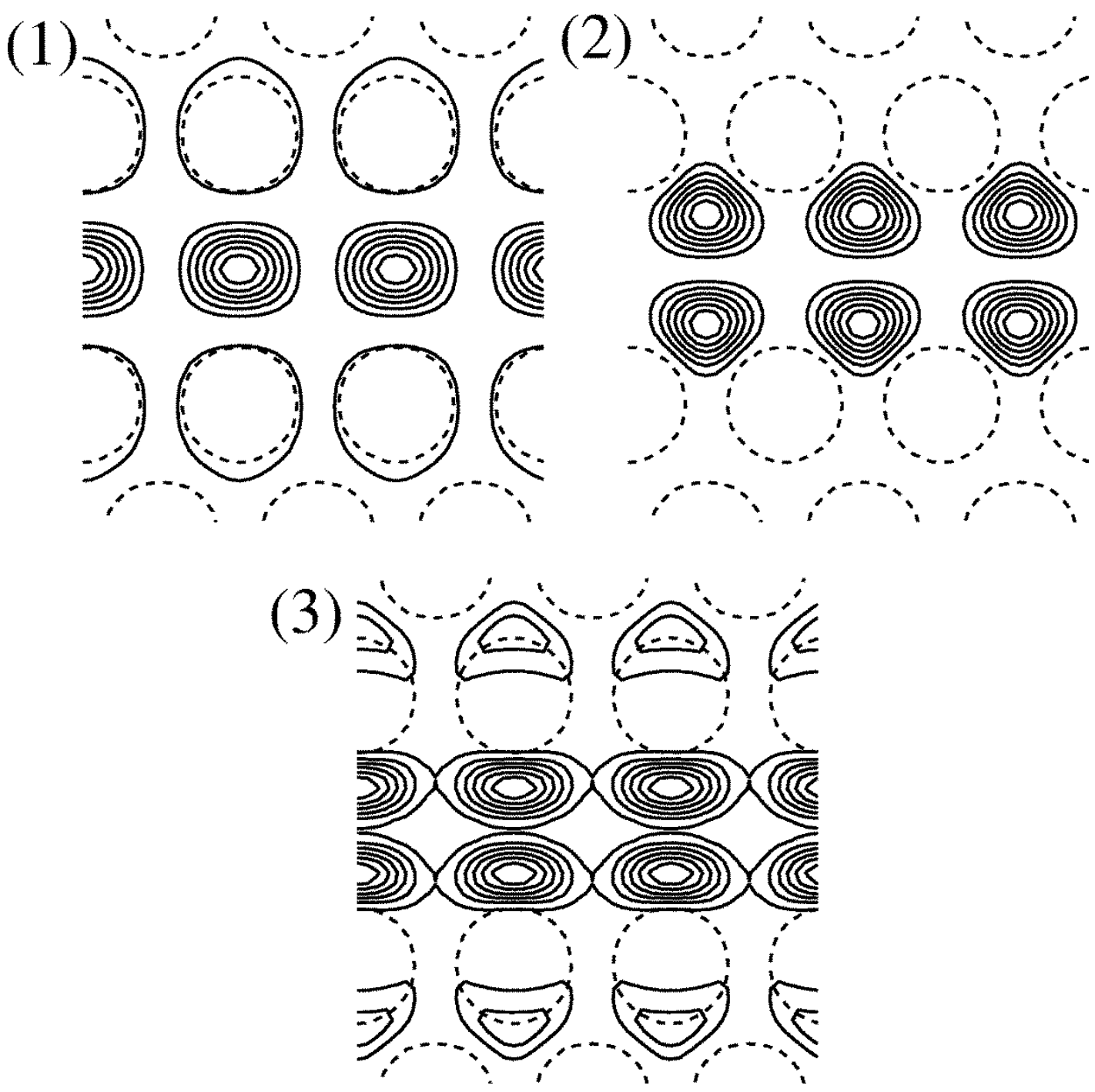

Fig. 7. Amplitude of magnetic field squared for the three bandgap-guided modes in the finite-height photonic crystal waveguide $(k \Lambda / 2 \pi=0.46)$.

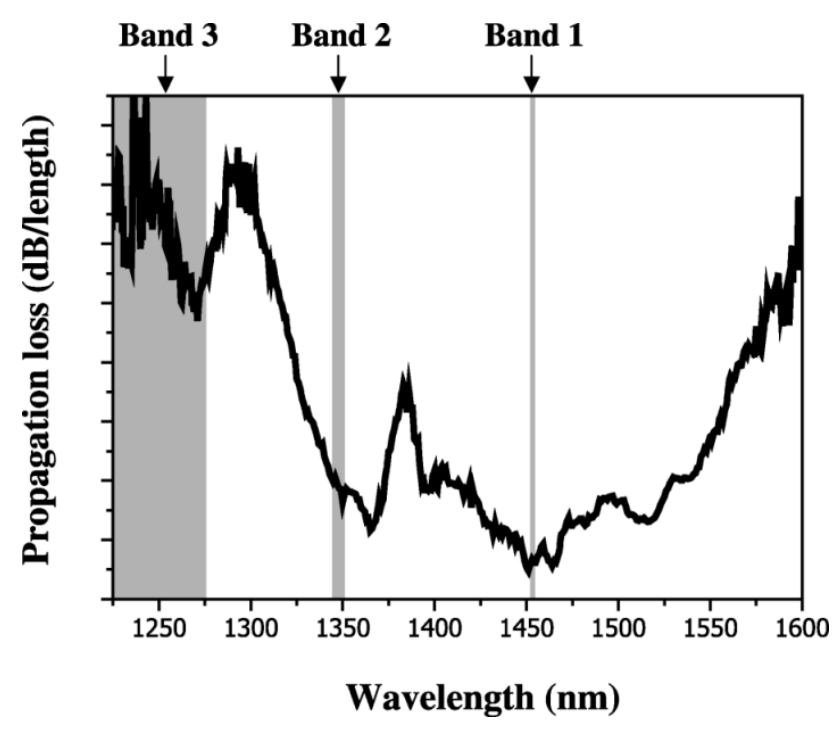

Fig. 8. Experimental propagation loss measurement for a SOI planar photonic crystal waveguide.

the center of the waveguide; this is not the case for the magnetic fields (see Fig. 7).
The localization of the first band in the bandgap is illustrated to the left in Fig. 9 (in field 1). The shaded region indicates the finite-height silicon slab. Outside the shaded region is the silica material above and below the silicon photonic crystal waveguide slab. From the contour plot, we see that the electric field is well localized. The localization is also studied by plotting the field along the dashed line and the dash-dot line, and we see that, outside the silicon slab, the field decays rapidly. This is also the case for the second band, where we also observed low experimental propagation losses. For the third band, however, the tails of the field extend far into the silica on either side of the silicon slab.

In the theoretical calculation, we assume that the silica on either side extends to infinity. It is necessary to assume that the structure is symmetric in this way because otherwise, the bandgap and leakage-free guided modes do not exist in the first place. For the case of fields 1 and 2 in Fig. 9, we conclude that the silica layer on top of the silicon photonic crystal slab in the experimental structure does not have to be thick compared with the height of the silicon slab. The silica layer on top has a thickness of approximately $50 \mathrm{~nm}$, which is roughly one-fifth of the height of the silicon slab. This is barely enough for fields 1 and 2 


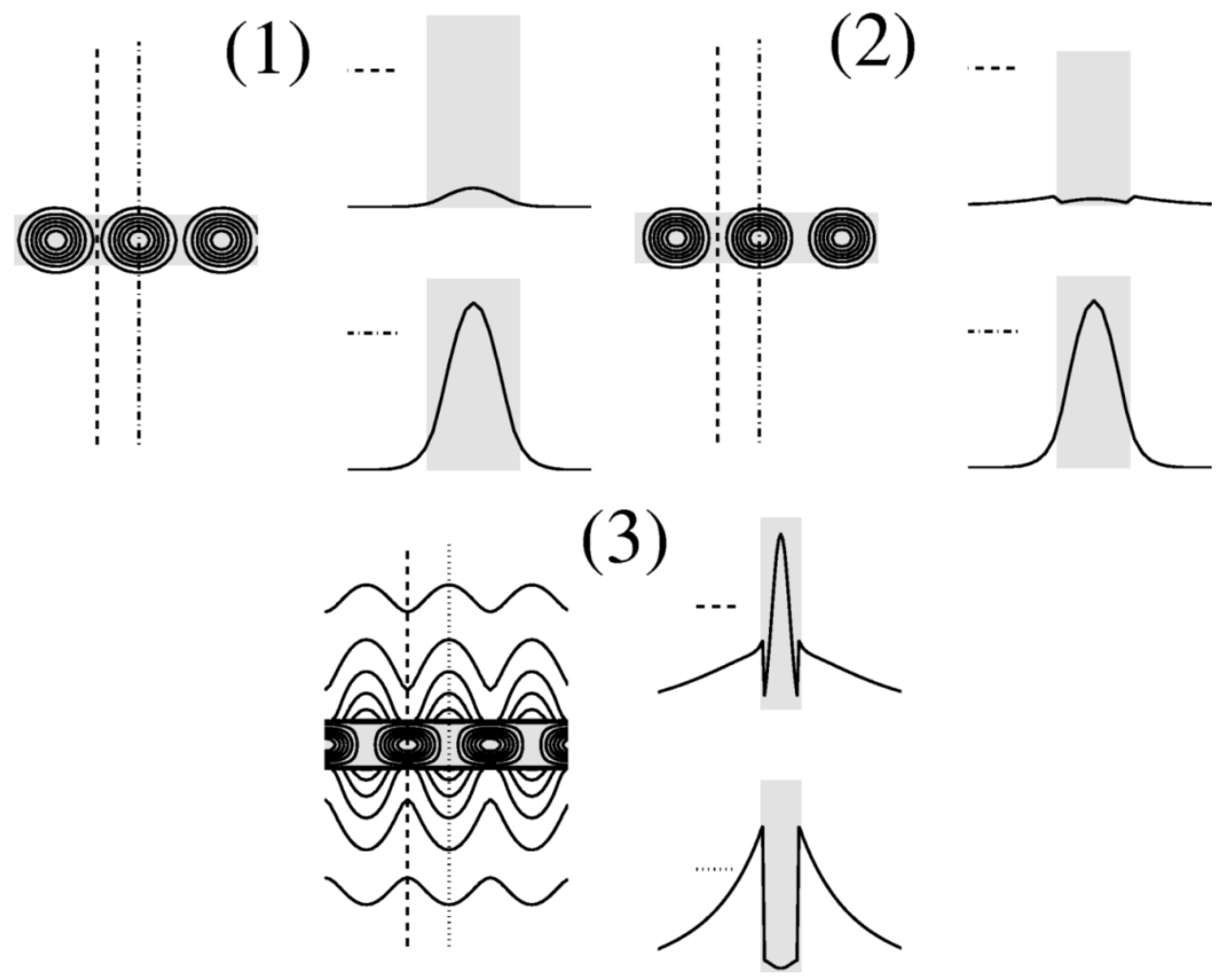

Fig. 9. Vertical cross section of the amplitude of electric field squared through the center of the finite height photonic crystal waveguide for the three bandgap-guided modes $(k \Lambda / 2 \pi=0.46)$.

to be only weakly affected by the fact that the real experimental structure is not symmetric. The top layer of silica is, however, not thick enough for the case of field 3 in Fig. 9. Accordingly, we expect that the third band in the bandgap will be stripped off.

The theoretical calculations suggest that guidance of light for the frequency range of the third band can be made possible by depositing a thick layer of silica (with air holes) on top of the structure. This might be worth pursuing because the calculated frequency range covered by the third band is very broad compared with the frequency ranges covered by bands 1 and 2 and because of the better impedance match for the modes of the third band to, e.g., a ridge waveguide.

\section{CONCLUSION}

In conclusion, design guidelines have been obtained for the size of holes in a finite-height 2-D photonic crystal waveguide by comparing 2-D calculations with dispersion relations for the media surrounding the waveguide. Three-dimensional calculations were compared to 2-D calculations, and we found that the number of guided modes changes and the frequencies of the modes are shifted. The distributions of the field for three guided modes calculated using 2-D and 3-D calculations were qualitatively similar. The analysis shows that when comparing theory and experimental results 3-D calculations are necessary to obtain good agreement.

The theoretically predicted frequency ranges, where the waveguide supports leakage-free guidance of light, were compared with experimental propagation loss measurements for the waveguide. Two out of three calculated frequency ranges coincided with low propagation losses, but the calculated frequency ranges were narrow compared with the measured frequency intervals with low propagation losses. This difference in width of frequency intervals can be due to variations in the experimental structure, e.g., fluctuations in the size and position of air holes. However, the difference can also be due to the fact that, although leakage-free guidance is not theoretically possible outside the calculated bands, the losses due to leakage of light are not necessarily high.

Low losses were not observed for the third calculated frequency range. The poor guidance for the third frequency range was explained theoretically by evaluating the vertical confinement of light for the bandgap-guided modes. The glass layer on top of the experimental structure is presently not thick enough for the structure to support guidance for the third frequency range.

\section{REFERENCES}

[1] E. Yablonovitch, "Inhibited spontaneous emission in solid-state physics and electronics," Phys. Rev. Lett., vol. 58, pp. 2059-2062, 1987.

[2] S. John, "Strong localization of photons in certain disordered dielectric superlattices," Phys. Rev. Lett., vol. 58, pp. 2486-2489, 1987.

[3] J. D. Joannopoulos, J. N. Winn, and R. D. Meade, Photonic Crystals: Molding the Flow of Light. Princeton, NJ: Princeton Univ. Press, 1995.

[4] T. F. Krauss and R. M. De La Rue, "Photonic crystals in the optical regime-Past, present and future," Prog. Quantum Electron., vol. 23, pp. 51-96, 1999.

[5] A. A. Erchak, D. J. Ripin, S. Fan, P. Rakich, J. D. Joannopoulos, E. P. Ippen, G. S. Petrich, and L. A. Kolodziejski, "Enhanced coupling to vertical radiation using a two-dimensional photonic crystal in a semiconductor light-emitting diode," Appl. Phys. Lett., vol. 78, pp. 563-565, 2001. 
[6] O. Painter, R. K. Lee, A. Scherer, A. Yariv, J. D. O'Brien, P. D. Dapkus, and I. Kim, "Two-dimensional photonic band-gap defect mode laser," Science-AAAS-Weekly Paper Edition, vol. 284, no. 5421, pp. 1819-1821, 1999.

[7] M. Meier, A. Dodabalapur, J. A. Rogers, R. E. Slusher, A. Mekis, and A. Timko, "Emission characteristics of two-dimensional organic photonic crystal lasers fabricated by replica modeling," J. Appl. Phys., vol. 86, pp. 3502-3507, 1999.

[8] J. Moosburger, M. Kamp, F. Klopf, J. P. Reithmaier, and A. Forchel, "Semiconductor lasers with 2-D-photonic crystal mirrors based on a wet-oxidized $\mathrm{Al}_{2} \mathrm{O}_{3}$-mask," IEEE Photon. Technol. Lett., vol. 13, pp. 406-408, May 2001.

[9] J. K. Hwang, H. Y. Ryu, D. S. Song, I. Y. Han, H. K. Park, D. H. Jang, and Y. H. Lee, "Continuous room-temperature operation of optically pumped two-dimensional photonic crystal lasers at $1.6 \mu \mathrm{m}$, , IEEE Photon. Technol. Lett., vol. 12, pp. 1295-1297, Oct. 2000.

[10] A. Mekis, J. C. Chen, I. Kurland, S. Fan, P. R. Villeneuve, and J. D. Joannopoulos, "High transmission through sharp bends in photonic crystal waveguides," Phys. Rev. Lett., vol. 77, pp. 3787-3790, 1996.

[11] T. Søndergaard and K. H. Dridi, "Energy flow in photonic crystal waveguides," Phys. Rev. B, Condens. Matter, vol. 61, pp. 15 688-15 696, 2000.

[12] H. Benisty, "Modal analysis of optical guides with two-dimensional photonic band-gap boundaries," J. Appl. Phys., vol. 79, pp. 7483-7492, 1996.

[13] A. Mekis, S. Fan, and J. D. Joannopoulos, "Bound states in photonic crystal waveguides and waveguide bends," Phys. Rev. B, Condens. Matter, vol. 58, pp. 4809-4817, 1998.

[14] E. Centeno and D. Felbacq, "Guiding waves with photonic crystals," Opt. Commun., vol. 160, pp. 57-60, 1999.

[15] R. W. Ziolkowski and M. Tanaka, "FDTD analysis of PBG waveguides, power splitters and switches," Opt. Quantum Electron., vol. 31, pp. 843-855, 1999.

[16] M. Bayindir, E. Ozbay, B. Temelkuran, M. M. Sigalas, C. M. Soukoulis, R. Biswas, and K. M. Ho, "Guiding, bending and splitting of electromagnetic waves in highly confined photonic crystal waveguides," Phys. Rev. B, Condens. Matter, vol. 63, p. 081 107, 2001

[17] A. Talneau, L. Le Gouezigou, and N. Bouadma, "Quantitative measurement of low propagation losses at $1.55 \mu \mathrm{m}$ on planar photonic crystal waveguides," Opt. Lett., vol. 26, no. 16, pp. 1259-1261, 2001.

[18] C. J. M. Smith, H. Benisty, S. Olivier, M. Rattier, C. Weisbuch, T. F. Krauss, R. M. De La Rue, R. Houdre, and U. Oesterle, "Low-loss channel waveguides with two-dimensional photonic crystal boundaries," Appl. Phys. Lett., vol. 77, pp. 2813-2815, 2000.

[19] S. Olivier, M. Rattier, H. Benisty, C. Weisbuch, C. J. M. Smith, R. M. De La Rue, T. F. Krauss, U. Oesterle, and R. Houdre, "Mini-stopbands of a one-dimensional system: The channel waveguide in a two-dimensional photonic crystal," Phys. Rev. B, vol. 63, p. 113 311, 2001.

[20] Ph. Lalanne and H. Benisty, "Out-of-plane losses of two-dimensional photonic crystals waveguides: Electromagnetic analysis," J. Appl. Phys., vol. 89, pp. 1512-1514, 2001.

[21] M. Palamaru and Ph. Lalanne, "Photonic crystal waveguides: Out-ofplane losses and adiabatic modal conversion," Appl. Phys. Lett., vol. 78, pp. 1466-1468, 2001

[22] T. Søndergaard, A. Bjarklev, M. Kristensen, J. Erland, and J. Broeng, "Designing finite height photonic crystal waveguides," Appl. Phys. Lett., vol. 77, pp. 785-787, 2000.

[23] T. Søndergaard, A. Bjarklev, J. Arentoft, M. Kristensen, J. Erland, J. Broeng, and S. E. B. Libori, "Designing finite-height photonic crystal waveguides: Confinement of light and dispersion relations," Opt. Comm., vol. 194, pp. 341-351, 2001

[24] S. Kuchinsky, D. C. Allan, N. F. Borelli, and J.-C. Cotteverte, "3D localization in a channel waveguide in a photonic crystal with 2D periodicity," Opt. Comm., vol. 175, pp. 147-152, 2000.

[25] A. Chutinan and S. Noda, "Waveguides and waveguide bends in twodimensional photonic crystal slabs," Phys. Rev. B, Condens. Matter, vol. 62, pp. 4488-4492, 2000

[26] S. G. Johnson, P. R. Villeneuve, S. Fan, and J. D. Joannopoulos, "Linear waveguides in photonic crystal slabs," Phys. Rev. B, Condens. Matter, vol. 62 , pp. 8212-8222, 2000.

[27] M. Loncar, T. Doll, J. Vuckovic, and A. Scherer, "Design and fabrication of silicon photonic crystal optical waveguides," J. Lightwave Technol., vol. 18 , pp. $1402-1411$, Oct. 2000
[28] M. Loncar, J. Vuckovic, and A. Scherer, "Methods for controlling positions of guided modes of photonic-crystal waveguides," J. Opt. Soc. Am. B., Opt. Phys., vol. 18, no. 9, pp. 1362-1368, 2001.

[29] M. D. B. Charlton, G. J. Parker, and M. E. Zoorob, "Recent developments in the design and fabrication of visible photonic band gap waveguide devices," J. Mater. Sci.-Mat. Electron., vol. 10, pp. 429-440, 1999.

[30] M. D. B. Charlton, M. E. Zoorob, G. J. Parker, M. C. Netti, J. J. Baumberg, S. J. Cox, and H. Kemhadjian, "Experimental investigation of photonic crystal waveguide devices and line-defect waveguide bends," Mater. Sci. Eng.: B, vol. 74, pp. 17-24, 2000.

[31] M. Loncar, D. Nedeljkovich, T. Doll, A. Vuckovic, A. Scherer, and T. P. Pearsall, "Waveguiding in planar photonic crystals," Appl. Phys. Lett., vol. 77, pp. 1937-1939, 2000.

[32] M. Tokushima, H. Kosaka, A. Tomita, and H. Yamada, "Lightwave propagation through a 120 degrees sharply bent single-line-defect photonic crystal waveguide," Appl. Phys. Lett., vol. 76, pp. 952-954, 2000.

[33] E. Chow, S. Y. Lin, J. R. Wendt, S. G. Johnson, and J. D. Joannopoulos, "Quantitative analysis of bending efficiency in photonic-crystal waveguide bends at $\lambda=1.55 \mathrm{~mm}$ wavelengths," Opt. Lett., vol. 26, pp. 286-288, 2001

[34] M. Notomi, A. Shinya, K. Yamada, J. Takahashi, C. Takahashi, and I. Yokohama, "Single-mode transmission within photonic bandgap of width-varied single-line-defect photonic crystal waveguides on SOI substrates," Electron. Lett., vol. 37, pp. 292-293, 2001.

[35] J. Arentoft, M. Kristensen, T. Søndergaard, and A. Boltasseva, "Realization of robust photonic crystal waveguides designed to reduce out-ofplane scattering," in Proc. 27th European Conf. Optical Communication (ECOC'01), vol. 4, Amsterdam, Paper th.a.2.6.

[36] J. Arentoft, T. Søndergaard, M. Kristensen, A. Boltasseva, M. Thorhauge, and L. Frandsen, "Low-loss silicon-on-insulator photonic crystal waveguides," Electron. Lett., vol. 38, pp. 274-275, 2002.

[37] R. D. Meade, A. M. Rappe, K. D. Brommer, J. D. Joannopoulos, and O. L. Alerhand, "Accurate theoretical analysis of photonic band-gap materials," Phys. Rev. B, Condens. Matter, vol. 48, no. 11, pp. 8434-8437, 1993.

Thomas Søndergaard received the M.Sc.E.E. degree from the Technical University of Denmark (DTU), Lyngby, in March 1999 and is pursuing the Ph.D. degree at DTU. His Ph.D. work contributes to two topics within optics of dielectric microstructures, namely, (spontaneous) emission of radiation by sources and planar photonic crystal waveguides.

Jesper Arentoft obtained the Masters of Science degree in chemistry at the University of Copenhagen, Copenhagen, Denmark, in 1996. The subject of the masters project was a theoretical/computational study of nonlinear optical properties of organic molecules.

From 1996 to 2000, during his Ph.D. study at COM/MIC, the Technical University of Denmark, Lyngby, he experimentally investigated nonlinear optical effects-including electrooptic effects and second harmonic generation-in silica glass waveguides induced by electric field poling. Following his Ph.D. study, he joined the planar photonic crystal project at COM. His work focuses on processing and optical characterization.

Martin Kristensen studied physics and chemistry and received the Ph.D. degree from University of Aarhus, Aarhus C, Denmark, in 1992.

After receiving the degree, he did research within quantum optics in Leiden, Holland. Since 1994, he worked with development of processes for fabrication of silica waveguides, UV writing of gratings and waveguides, poling of silica-based waveguides, and development of photonic crystal waveguides. $\mathrm{He}$ is Research Professor in glass at COM, the Technical University of Denmark, Lyngby. 